\title{
THE GENERALIZED $t$-COMTET NUMBERS AND SOME COMBINATORIAL APPLICATIONS
}

\author{
HACÈNE BELBACHIR, CAROLINA FORERO, AND JOSÉ L. RAMÍREZ
}

Received 22 February, 2018

\begin{abstract}
In the present article we use a combinatorial approach to generalize the Comtet numbers. In particular, we establish some combinatorial identities, recurrence relations and generating functions. Additionally, for some particular cases we study their relationship with $t$ successive associated Stirling numbers and their $q$-analogue.
\end{abstract}

2010 Mathematics Subject Classification: 11B83; 11B73; 05A15; 05A19

Keywords: Stirling numbers of the second kind, $t$-successive associated Stirling numbers, Comtet numbers, combinatorial identities

\section{INTRODUCTION}

It is well-known that the Stirling numbers of the second kind $\left\{\begin{array}{l}n \\ k\end{array}\right\}$ count the number of partitions of a set with $n$ elements into $k$ non-empty blocks. This sequence satisfies the recurrence relation

$$
\left\{\begin{array}{l}
n \\
k
\end{array}\right\}=k\left\{\begin{array}{c}
n-1 \\
k
\end{array}\right\}+\left\{\begin{array}{l}
n-1 \\
k-1
\end{array}\right\},
$$

with the initial conditions $\left\{\begin{array}{l}0 \\ 0\end{array}\right\}=1$ and $\left\{\begin{array}{l}n \\ 0\end{array}\right\}=\left\{\begin{array}{l}0 \\ n\end{array}\right\}=0$.

The Stirling numbers $\left\{\begin{array}{l}n \\ k\end{array}\right\}$ can be generalized to the associated Stirling numbers of the second kind $\left\{\begin{array}{l}n \\ k\end{array}\right\}_{\geq m}$ (cf. [1, 4, 7, 8, 10,11, 18, 20])) by means of a restriction on the size of the blocks. In particular, this sequence gives the number of partitions of $n$ elements into $k$ blocks, such that each block contains at least $m$ elements. It is clear that $\left\{\begin{array}{l}n \\ k\end{array}\right\}_{>1}=\left\{\begin{array}{l}n \\ k\end{array}\right\}$. This combinatorial sequence has been applied to the study of some special polynomials such as generalized Bernoulli and Cauchy polynomials, (see, e.g., [12-16]).

Recently, Belbachir and Tebtoub [2] considered a variation for the associated Stirling numbers. They introduced the 2-successive associated Stirling numbers of the second kind $\left\{\begin{array}{l}n \\ k\end{array}\right\}^{[2]}$. This new sequence counts the number of partitions of $n$ elements

The research of José L. Ramírez was partially supported by Universidad Nacional de Colombia, Project No. 37805. 
into $k$ blocks, with the additional condition that each block contains at least two consecutive elements. Moreover, the last element $n$ must either form a block with its predecessor or belong to another block satisfying the previous conditions. In [2], the authors derived the recurrence

$$
\left\{\begin{array}{l}
n \\
k
\end{array}\right\}^{[2]}=k\left\{\begin{array}{c}
n-1 \\
k
\end{array}\right\}^{[2]}+\left\{\begin{array}{l}
n-2 \\
k-1
\end{array}\right\}^{[2]}, n \geq 2 k,
$$

with the initial conditions $\left\{\begin{array}{c}0 \\ 0\end{array}\right\}^{[2]}=1,\left\{\begin{array}{c}n \\ n-1\end{array}\right\}^{[2]}=0$ and $\left\{\begin{array}{l}n \\ 0\end{array}\right\}=0$ for $n \geq 1$.

Inspired by these results, in this paper we aim to investigate the sequence $\left\{a^{[t]}(n, k)\right\}_{n, k \geq 0}$, defined by the recurrence relation

$$
a^{[t]}(n, k)=u_{k} a^{[t]}(n-1, k)+a^{[t]}(n-t, k-1), \quad n \geq t k,
$$

with the initial conditions $a^{[t]}(0,0)=1, a^{[t]}(n, n-\ell)=0$ for $\ell=1,2, \ldots, t-1$ and $a^{[t]}(n, 0)=0$, for $n \geq 1$. Moreover, $\left\{u_{n}\right\}$ is a sequence of real numbers.

We will call the sequence $\left\{a^{[t]}(n, k)\right\}_{n, k \geq 0}$ the generalized $t$-Comtet numbers. The reason for this name is that for $t=1$ we recover the Comtet numbers (see, e.g., $[9,21])$. Note that if $u_{k}=k$, then $a^{[t]}(n, k)=\left\{\begin{array}{l}n \\ k\end{array}\right\}^{[t]}$. This sequence is called by Belbachir and Tebtoub [3] as the $t$-successive associated Stirling numbers. If $t=2$ and $u_{k}=k$, then $a^{[2]}(n, k)=\left\{\begin{array}{l}n \\ k\end{array}\right\}^{[2]}$. If $t=1$ and $u_{k}=k$, then $a^{[1]}(n, k)=\left\{\begin{array}{l}n \\ k\end{array}\right\}$.

In this paper our goal is to give the recurrence relation, the generating function and some combinatorial identities. For some particular cases, we give combinatorial interpretations.

\section{BASIC PROPERTIES}

From the recurrence relation (1.1) we obtain the following generating function.

Theorem 1. For $k \geq 1$,

$$
A_{k}^{[t]}(x):=\sum_{n \geq t k} a^{[t]}(n, k) x^{n}=\frac{x^{t k}}{\left(1-u_{0} x\right)\left(1-u_{1} x\right)\left(1-u_{2} x\right) \cdots\left(1-u_{k} x\right)},
$$

with $A_{0}^{[t]}(x)=\frac{1}{1-u_{0} x}$.

Proof. Multiplying both sides of (1.1) by $x^{n}$ and summing over $n \geq t k$, we have

$$
\begin{aligned}
A_{k}^{[t]}(x) & =u_{k} \sum_{n \geq t k} a^{[t]}(n-1, k) x^{n}+\sum_{n \geq t k} a^{[t]}(n-t, k-1) x^{n} \\
& =u_{k} x \sum_{n \geq t k} a^{[t]}(n, k) x^{n}+\sum_{n \geq t k-t} a^{[t]}(n, k-1) x^{n+t} \\
& =u_{k} x A_{k}^{[t]}(x)+x^{t} A_{k-1}^{[t]}(x) .
\end{aligned}
$$


Then

$$
A_{k}^{[t]}(x)=\frac{x^{t} A_{k-1}^{[t]}(x)}{1-u_{k} x} .
$$

Iterating this last recurrence, we obtain (2.1).

From the above relation, we have the following combinatorial expression.

Corollary 1. The generalized $t$-Comtet numbers are given by the explicit identity

$$
a^{[t]}(n, k)=\sum_{i_{1}+i_{2}+\cdots+i_{k}=n-t k} u_{1}^{i_{1}} u_{2}^{i_{2}} \cdots u_{k}^{i_{k}},
$$

for $n \geq t k$.

Theorem 2. The generalized t-Comtet numbers satisfy the following recurrence relation

$$
a^{[t]}(n, k)=\sum_{i=0}^{n-t k} u_{k}^{i} a^{[t]}(n-i-t, k-1) .
$$

Proof. For $n \geq t k$,

$$
\begin{aligned}
& a(n, k) \quad=u_{k} a(n-1, k) \quad+a(n-t, k-1), \\
& u_{k} a(n-1, k) \quad=u_{k}^{2} a(n-2, k) \quad+u_{k} a(n-1-t, k-1) \text {, } \\
& u_{k}^{2} a(n-2, k) \quad=u_{k}^{3} a(n-3, k) \quad+u_{k}^{2} a(n-2-t, k-1) \text {, } \\
& u_{k}^{n-t k-1} a(t k+1, k)=u_{k}^{n-t k} a(t k, k) \quad+u_{k}^{n-t k-1} a(t k+1-t, k-1), \\
& u_{k}^{n-t k} a(t k, k)=u_{k}^{n-t k+1} a(t k-1, k)+u_{k}^{n-t k} a(t(k-1), k-1) \text {, }
\end{aligned}
$$

by summing, we get the result.

Theorem 3. We have the following rational explicit formula

$$
a^{[t]}(n+t k, k)=\sum_{j=0}^{k} \frac{u_{j}^{k+n}}{\prod_{i \neq j}\left(u_{j}-u_{i}\right)},
$$

which is independent from $t$.

Proof. We have

$$
A_{k}^{[t]}(x)=\sum_{n \geq t k} a^{[t]}(n, k) x^{n}=x^{t k} \sum_{n \geq 0} a^{[t]}(n+t k, k) x^{n},
$$


then

$$
\begin{aligned}
\sum_{n \geq 0} a^{[t]}(n+t k, k) x^{n} & =\frac{1}{\left(1-u_{0} x\right)\left(1-u_{1} x\right) \cdots\left(1-u_{k} x\right)} \\
& =\sum_{j=0}^{k} \frac{\alpha_{j}}{1-u_{j} x} \\
& =\sum_{j=0}^{k} \frac{u_{j}^{k}}{\prod_{i \neq j}\left(u_{j}-u_{i}\right)} \sum_{n \geq 0} u_{j}^{n} x^{n} \\
& =\sum_{n \geq 0}\left(\sum_{j=0}^{k} \frac{u_{j}^{k+n}}{\prod_{i \neq j}\left(u_{j}-u_{i}\right)}\right) x^{n},
\end{aligned}
$$

which gives the result.

Corollary 2. The dual expression depending on $t$

$$
a^{[t]}(n, k)=\sum_{j=0}^{k} \frac{u_{j}^{n+k(t-1)}}{\prod_{i \neq j}\left(u_{j}-u_{i}\right)} .
$$

\subsection{Exponential generating function for the $t$-Comtet numbers}

Let $u_{1}, \ldots, u_{k}$ be a sequence of complex numbers and let $\left(A_{m}\right)_{m=1, \ldots, n}$ be the sequence of matrices such that $A_{m}$ is $m \times m$-matrix

$$
A_{m}=\left[\begin{array}{cccc}
u_{k-m} & u_{k-m+1} & \cdots & u_{k-1} \\
u_{k-m+1} & \cdots & & u_{k} \\
\vdots & \ddots & \ddots & 0 \\
u_{k-1} & u_{k} & 0 & 0
\end{array}\right]
$$

with the convention that $u<0=0$.

Consider also

$$
\sigma_{j}=(-1)^{j} \sum_{1 \leq k_{1}<k_{2}<\cdots<k_{j} \leq k} u_{k_{1}} \cdots u_{k_{j}},
$$

(the alternate sequence of elementary symmetric function associated to $u_{1}, u_{2}, \ldots, u_{k}$ ).

We have $\left(p-u_{1}\right)\left(p-u_{2}\right) \cdots\left(p-u_{k}\right)=p^{k}+\sigma_{1} p^{k-1}+\sigma_{2} p^{k-2}+\cdots+\sigma_{k}$. Now we can state the following lemma which will be used to establish the main result of this subsection.

Lemma 1. We have the following decomposition

$$
\frac{1}{p^{n}\left(p-u_{1}\right) \cdots\left(p-u_{k}\right)}=\sum_{i=0}^{n} \frac{\alpha_{n-i}}{p^{i}}+\sum_{j=1}^{k} \frac{\beta_{j}}{p-u_{j}},
$$




$$
\text { with } \alpha_{i}=\frac{(-1)^{\lfloor(i+1) / 2\rfloor}}{\sigma_{k}^{i+1}} \operatorname{det}\left(A_{i}\right), \alpha_{0}=1 / \sigma_{k} \text {, and } \beta_{j}=\frac{1}{u_{j}^{n} \prod_{\substack{i=1 \\ i \neq j}}^{k}\left(u_{j}-u_{i}\right)} \text {. }
$$

Proof. We leave the proof to the reader.

$$
\begin{aligned}
\text { Let } C_{k}^{[t]}(x):=\sum_{n \geq t k} a^{[t]}(n, k) \frac{x^{n}}{n !}, & \text { with } C_{0}^{[t]}(x)=1 \text {. We have } \\
\frac{\partial^{t}}{\partial x^{t}} C_{k}^{[t]}(x) & =\sum_{n \geq t(k-1)} a^{[t]}(n+t, k) \frac{x^{n}}{n !},
\end{aligned}
$$

which gives using relation (1.1),

$$
\frac{\partial^{t}}{\partial x^{t}} C_{k}^{[t]}(x)=u_{k} \frac{\partial^{t-1}}{\partial x^{t-1}} C_{k}^{[t]}(x)+C_{k-1}^{[t]}(x) .
$$

To solve the linear recurrence differential equation we use Laplace transform. Using the fact that

$$
C_{k}^{[t]}(0)=\left.\frac{\partial}{\partial x} C_{k}^{[t]}(y)\right|_{y=0}=\cdots=\left.\frac{\partial^{t-1}}{\partial x^{t-1}} C_{k}^{[t]}(y)\right|_{y=0}=0,
$$

we get

$$
\prod_{i=1}^{k}\left(p^{t}-u_{i} p^{t-1}\right) \mathscr{L}\left(C_{k}^{[t]}(y)\right)=\mathscr{L}\left(C_{k-1}^{[t]}\right),
$$

where $\mathscr{L}\left(C_{k}^{[t]}(y)\right)=\int_{0}^{\infty} C_{k}^{[t]} e^{p y} \partial y$.

Thus by recursion, we get

$$
p^{(t-1) k} \prod_{i=1}^{k}\left(p-u_{i}\right) \mathscr{L}\left(C_{k}^{[t]}(y)\right)=\mathscr{L}\left(C_{0}^{[t]}(y)\right)=\mathscr{L}(u(y)),
$$

where $u(t)$ is the Heaviside function. Using Lemma 1, we have

$$
\left.\mathscr{L}\left(C_{k}^{[t]}(y)\right)\right)=\mathscr{L}(u(y))\left[\sum_{i=0}^{(t-1) k} \frac{\alpha_{i}}{p^{i}}+\sum_{j=1}^{k} \frac{\beta_{j}}{p-u_{j}}\right] .
$$

The inverse Laplace transform gives,

$$
C_{k}^{[t]}(y)=\sum_{i=1}^{(t-1) k} \alpha_{i} \frac{y^{i-1}}{(i-1) !}+\sum_{j=1}^{k} \beta_{j} e^{u_{j} y}
$$


Theorem 4. The exponential generating function of $t$-Comtet numbers is given by

$$
\sum_{n \geq t k} a^{[t]}(n, k) \frac{x^{n}}{n !}=\sum_{i=1}^{(t-1) k} \alpha_{i} \frac{x^{i-1}}{(i-1) !}+\sum_{j=1}^{k} \beta_{j} e^{u_{j} x} .
$$

\section{THE 2-SUCCESSIVE ASSOCIATED $r$-WHITNEY NUMBERS}

In this section, we study the particular case $u_{k}=k m+r$. Let $n, r \geq 0$ be integers. Let $\Pi_{r}(n, k)$ denote the set of partitions of the set $[n+r]:=\{1, \ldots, n, n+1, \ldots, n+$ $r$ \} into $k+r$ blocks, such that, the first $r$ elements are in distinct blocks. The elements $\{1,2, \ldots, r\}$ will be called special elements. A block of a partition of the above set is called special if it contains special element. The cardinality of $\Pi_{r}(n, k)$ is the $r$-Stirling numbers of the second kind [5].

The 2-successive associated $r$-Whitney numbers of the second kind, denoted $W_{m, r}^{[2]}(n, k)$, count the number of partitions in $\Pi_{r}(n, k)$, such that:

- the $k$ non-special blocks contain at least two consecutive numbers,

- all the elements but the last one and its predecessor in non-special blocks are coloured with one of $m$ colours independently,

- the elements in the special blocks are not coloured,

- the last element $n+r$ must either form a block with its predecessor or belong to another block (special or not-special) satisfying the previous conditions.

We denote by $\Pi_{r, m}^{[2]}(n, k)$ the set of partitions in $\Pi_{r}(n, k)$ that satisfying the previous conditions. It is clear that if $r=0$ and $m=1$, then $W_{1,0}^{[2]}(n, k)=\left\{\begin{array}{l}n \\ k\end{array}\right\}^{[2]}$, (see [2]).

For example, $W_{2,3}^{[2]}(5,2)=15$ with the partitions being (the $m=2$ different colours of the elements will be fixed as red and blue, and the $r=3$ special elements are $\overline{1}, \overline{2}$ and $\overline{3}$ ):

$$
\begin{array}{rr}
\{\{\overline{1}\},\{\overline{2}\},\{\overline{3}\},\{4,5,6\},\{7,8\}\}, & \{\{\overline{1}\},\{\overline{2}\},\{\overline{3}\},\{4,5,6\},\{7,8\}\}, \\
\{\{\overline{1}, 6\},\{\overline{2}\},\{\overline{3}\},\{4,5\},\{7,8\}\}, & \{\{\overline{1}\},\{\overline{2}, 6\},\{\overline{3}\},\{4,5\},\{7,8\}\}, \\
\{\{\overline{1}\},\{\overline{2}\},\{\overline{3}, 6\},\{4,5\},\{7,8\}\}, & \{\{\overline{1}, 4\},\{\overline{2}\},\{\overline{3}\},\{5,6\},\{7,8\}\}, \\
\{\{\overline{1}\},\{\overline{2}, 4\},\{\overline{3}\},\{5,6\},\{7,8\}\}, & \{\{\overline{1}\},\{\overline{2}\},\{\overline{3}, 4\},\{5,6\},\{7,8\}\}, \\
\{\{\overline{1}, 8\},\{\overline{2}\},\{\overline{3}\},\{4,5\},\{6,7\}\}, & \{\{\overline{1}\},\{\overline{2}, 8\},\{\overline{3}\},\{4,5\},\{6,7\}\}, \\
\{\{\overline{1}\},\{\overline{2}\},\{\overline{3}, 8\},\{4,5\},\{6,7\}\}, & \{\{\overline{1}\},\{\overline{2}\},\{\overline{3}\},\{4,5,8\},\{6,7\}\}, \\
\{\{\overline{1}\},\{\overline{2}\},\{\overline{3}\},\{4,5,8\},\{6,7\}\}, & \{\{\overline{1}\},\{\overline{2}\},\{\overline{3}\},\{4,5\},\{6,7,8\}\}, \\
\{\{\overline{1}\},\{\overline{2}\},\{\overline{3}\},\{4,5\},\{6,7,8\}\} .
\end{array}
$$

Theorem 5. For $n \geq 2 k$, we have

$$
W_{m, r}^{[2]}(n, k)=(k m+r) W_{m, r}^{[2]}(n-1, k)+W_{m, r}^{[2]}(n-2, k-1) .
$$


Proof. For any set partition of $\Pi_{r, m}^{[2]}(n, k)$, there are three options: either $n+r$ form a block with its predecessor $(n+r-1)$, or $n+r$ is in a special block or $n+r$ is in a non-special block. In the first case, there are $W_{m, r}^{[2]}(n-2, k-1)$ possibilities. In the second case, the element $n+r$ can be place into one of the $r$ special blocks and the remaining elements can be chosen in $W_{m, r}^{[2]}(n-1, k)$. Altogether, we have $r W_{m, r}^{[2]}(n-1, k)$ possibilities. For the third case, we can follow a similar argument, then we obtain $k m W_{m, r}^{[2]}(n-1, k)$ possibilities.

A comparison of (3.1) and (1.1) shows that $a^{[2]}(n, k)=W_{m, r}^{[2]}(n, k)$ for $u_{k}=k m+$ $r$. Therefore, from Theorem 1 and Corollary 1 we get the following corollaries.

Corollary 3. For $k \geq 1$,

$$
\begin{aligned}
W_{k}^{[2]}(x):= & \sum_{n \geq 2 k} W_{m, r}^{[2]}(n, k) x^{n} \\
& =\frac{x^{2 k}}{(1-r x)(1-(m+r) x)(1-(2 m+r) x) \cdots(1-(k m+r) x)},
\end{aligned}
$$

with $W_{0}^{[2]}(x)=\frac{1}{1-r x}$. Moreover, the 2-successive associated $r$-Whitney numbers of the second kind are given by the explicit identity

$$
W_{m, r}^{[2]}(n, k)=\sum_{i_{0}+i_{1}+i_{2}+\cdots+i_{k}=n-2 k} r^{i_{0}}(m+r)^{i_{1}} \cdots(k m+r)^{i_{k}},
$$

for $n \geq 2 k$.

In particular, for $m=1$ and $r=0$ we obtain the generating function of the 2successive associated Stirling numbers of the second kind. $k \geq 1$,

Corollary 4. (see [2, Theorem 2.3 and Corollary 2.4] and [3, Theorem 18]) For

$$
A_{k}(x):=\sum_{n \geq 2 k}\left\{\begin{array}{l}
n \\
k
\end{array}\right\}^{[2]} x^{n}=\frac{x^{2 k}}{(1-x)(1-2 x) \cdots(1-k x)},
$$

with $A_{0}(x)=1$. Moreover,

$$
\left\{\begin{array}{l}
n \\
k
\end{array}\right\}^{[2]}=\sum_{i_{1}+i_{2}+\cdots+i_{k}=n-2 k} 1^{i_{1}} 2^{i_{2}} \cdots k^{i_{k}} .
$$

Our next identity expresses $W_{m, r}^{[2]}(n, k)$ in terms of $\left\{\begin{array}{c}i \\ k\end{array}\right\}^{[2]}$ for $i \leq n$. 
Theorem 6. Let $n, k \geq 0$,

$$
W_{m, r}^{[2]}(n, k)=\sum_{i=2 k}^{n} r^{n-i}\left(\begin{array}{l}
n-k \\
n-i
\end{array}\right) m^{i-2 k}\left\{\begin{array}{l}
i \\
k
\end{array}\right\}^{[2]} .
$$

Proof. From (3.2) we have

$$
\begin{aligned}
\sum_{n \geq 2 k} W_{m, r}^{[2]}(n, k) x^{n} & =\frac{x^{2 k}}{(1-r x)(1-(m+r) x)(1-(2 m+r) x) \cdots(1-(k m+r) x)} \\
& =\frac{x^{2 k}}{(1-r x)^{k+1}\left(1-\frac{m x}{1-r x}\right)\left(1-\frac{2 m x}{1-r x}\right) \cdots\left(1-\frac{k m x}{1-r x}\right)} \\
& =\frac{(1-r x)^{k-1}}{m^{2 k}} \frac{\left(\frac{m x}{1-r x}\right)^{2 k}}{\left(1-\frac{m x}{1-r x}\right)\left(1-\frac{2 m x}{1-r x}\right) \cdots\left(1-\frac{k m x}{1-r x}\right)} \\
& =\frac{(1-r x)^{k-1}}{m^{2 k}} \frac{y^{2 k}}{(1-y)(1-2 y) \cdots(1-k m y)},
\end{aligned}
$$

where $y=\frac{m x}{1-r x}$.

Therefore from (3.4), we have

$$
\begin{aligned}
& \sum_{n \geq 2 k} W_{m, r}^{[2]}(n, k) x^{n}=\frac{(1-r x)^{k-1}}{m^{2 k}} \sum_{i \geq 2 k}\left\{\begin{array}{l}
i \\
k
\end{array}\right\}^{[2]} y^{i} \\
& =\sum_{i \geq 2 k}\left\{\begin{array}{l}
i \\
k
\end{array}\right\}^{[2]} \frac{m^{i-2 k} x^{i}}{(1-r x)^{i-k+1}} \\
& =\sum_{i \geq 2 k} \sum_{j \geq 0} m^{i-2 k}\left(\begin{array}{c}
i-k+j \\
i-k
\end{array}\right) r^{j}\left\{\begin{array}{l}
i \\
k
\end{array}\right\}^{[2]} x^{i+j} .
\end{aligned}
$$

Comparing the coefficients of $x^{n}$, we obtain (3.5).

Combinatorial proof: We can construct any set partition of $\Pi_{r, m}^{[2]}(n, k)$ as follows: we put $n-i$ elements in the special blocks. Then there are $\left(\begin{array}{c}n-k \\ i-k\end{array}\right) r^{n-i}$ possibilities. Note that we have to subtract $k$ elements of $n$ because in the non-special blocks there are at least two consecutive numbers. The remaining $i$ elements $(i \geq 2 k)$ can be chosen in $m^{i-2 k}\left\{\begin{array}{l}i \\ k\end{array}\right\}$ ways. The factor $m^{i-2 k}$ accounts for the $i-2 k$ non-minimal elements within these blocks that are each to be colored in one of $m$ ways.

From Theorem 5 and by induction on $n$ we obtain the following identity. 
Theorem 7. For $n \geq 2 k$ we have

$$
W_{m, r}^{[2]}(n, k)=\frac{1}{m^{k} k !} \sum_{j=0}^{k}(-1)^{k-j}\left(\begin{array}{l}
k \\
j
\end{array}\right)(m j+r)^{n-k} .
$$

Proof. Let,

$$
\begin{aligned}
W_{m, r}^{[2]}(n, k)= & \frac{(m k+r)}{m^{k} k !} \sum_{j=0}^{k}(-1)^{k-j}\left(\begin{array}{l}
k \\
j
\end{array}\right)(m j+r)^{n-1-k} \\
& +\frac{1}{m^{k-1} k !} \sum_{j=0}^{k}(-1)^{k-1-j}\left(\begin{array}{c}
k-1 \\
j
\end{array}\right)(m j+r)^{n-1-k} \\
= & \frac{(m k+r)}{m^{k-1} k !} \sum_{j=1}^{k}(-1)^{k-j}\left(\begin{array}{c}
k-1 \\
j-1
\end{array}\right)(m j+r)^{n-1-k} \\
& +\frac{r}{m^{k} k !} \sum_{j=0}^{k}(-1)^{k-j}\left(\begin{array}{l}
k \\
j
\end{array}\right)(m j+r)^{n-1-k} \\
= & \frac{1}{m^{k} k !} \sum_{i=0}^{n-1-k} r^{n-1-k-i} m^{i}\left[\begin{array}{l}
k \\
j=0
\end{array}(-1)^{k-j} j^{i+1}\left(\begin{array}{l}
k \\
j
\end{array}\right) m\right. \\
& \left.+\sum_{j=0}^{k}(-1)^{k-j} j^{i} r\left(\begin{array}{l}
k \\
j
\end{array}\right)\right] \\
= & \frac{1}{m^{k} k !} \sum_{j=0}^{k}(-1)^{k-j}\left(\begin{array}{l}
k \\
j
\end{array}\right)(m j+r)^{n-k}
\end{aligned}
$$

\subsection{Relations with the $r$-Whitney numbers}

The $r$-Whitney numbers of the second kind $W_{m, r}(n, k)$ were defined by Mezó [17] as the connecting coefficients between some particular polynomials.

For non-negative integers $n, k$ and $r$ with $n \geq k \geq 0$ and for any integer $m>0$

$$
(m x+r)^{n}=\sum_{k=0}^{n} m^{k} W_{m, r}(n, k) x^{\underline{k}},
$$

where $x^{\underline{n}}=x(x-1) \cdots(x-n+1)$ for $n \geq 1$, and $x^{\underline{0}}=1$.

The $r$-Whitney numbers of the second kind satisfy the recurrence [17]

$$
W_{m, r}(n, k)=W_{m, r}(n-1, k-1)+(k m+r) W_{m, r}(n-1, k) .
$$


Comparing (3.7) and (3.1) we have the following relation.

Corollary 5. [2, Theorem 4.1] For $n \geq 2 k$,

$$
W_{m, r}^{[2]}(n, k)=W_{m, r}(n-k, k) .
$$

Mezô and Ramírez [19] studied the $r$-Whitney matrices of the second and the first kind and they derived several identities for these matrices. In particular, the $r$-Whitney matrix of the second kind is defined by

$$
\left[W_{m, r}(n, k)\right]_{n, k \geq 0}=\left[\begin{array}{ccccc}
1 & 0 & 0 & 0 & 0 \\
r & 1 & 0 & 0 & 0 \\
r^{2} & m+2 r & 1 & 0 & 0 \\
r^{3} & m^{2}+3 r m+3 r^{2} & 3 m+3 r & 1 & 0 \\
r^{4} & m^{3}+4 r m^{2}+6 r^{2} m+4 r^{3} & 7 m^{2}+12 r m+6 r^{2} & 6 m+4 r & 1 \\
\vdots & & \vdots & & \vdots
\end{array}\right] .
$$

Notice that the sequence $\left(W_{m, r}^{[2]}(n, k)\right)_{k}$ corresponds with the sequence of elements on rays in direction $(1,1)$ over the $r$-Whitney matrix of the second kind.

\section{THE $t$-SUCCESSIVE ASSOCIATED $r$-WHITNEY NUMBERS}

In this section, we consider the rays in direction $(s, 1)$, i.e., we are going to study the sequence $\left\{W_{m, r}(n-s k, k)\right\}$. We denote by $W_{m, r}^{[t]}(n, k)$ the number $W_{m, r}(n-$ $s k, k)$, where $t=s+1$. We call this new sequence the $t$-successive associated $r$ Whitney numbers of the second kind. It is possible to show that the $t$-successive associated $r$-Whitney numbers count the number of partitions in $\Pi_{r}(n, k)$, such that:

- the $k$ non-special blocks contain at least $t$ consecutive numbers,

- all the elements but the last one and its $t-1$ predecessors in non-special blocks are coloured with one of $m$ colours independently,

- the elements in the special blocks are not coloured,

- the last element $n+r$ must either form a block with its $t$-1-predecessors or belong to another block (special or not-special) satisfying the previous conditions.

Reasoning in a similar manner as in Theorem 5 we obtain the following results.

Theorem 8. For $n \geq t k$, we have

$$
W_{m, r}^{[t]}(n, k)=(k m+r) W_{m, r}^{[t]}(n-1, k)+W_{m, r}^{[t]}(n-t, k-1) .
$$

For $k \geq 1$,

$$
\begin{aligned}
W_{k}^{[t]}(x):= & \sum_{n \geq 0} W_{m, r}^{[t]}(n, k) x^{n} \\
& =\frac{x^{t k}}{(1-r x)(1-(m+r) x)(1-(2 m+r) x) \cdots(1-(k m+r) x)},
\end{aligned}
$$


with $W_{0}^{[t]}(x)=\frac{1}{1-r x}$. Moreover, for $n \geq t k$ we have

$$
W_{m, r}^{[t]}(n, k)=\frac{1}{m^{k} k !} \sum_{j=0}^{k}(-1)^{k-j}\left(\begin{array}{l}
k \\
j
\end{array}\right)(m j+r)^{n-(t-1) k} .
$$

As corollary for $t=2$ we get [3, Theorem 4, Theorem 6 and Theorem 7]. It is not difficult to generalize the relation given in Theorem 6 .

Theorem 9. If $n, k \geq 0$, then

$$
W_{m, r}^{[t]}(n, k)=\sum_{i=t k}^{n} r^{n-i}\left(\begin{array}{c}
n-k \\
n-i
\end{array}\right) m^{i-t k}\left\{\begin{array}{l}
i \\
k
\end{array}\right\}^{[t]} .
$$

Consequence. From Equation (4.2) we deduce that $W_{m, r}^{[t]}(n+(t-1) k, k)$ are the classical $r$-Whitney numbers $W_{m, r}(n, k)$.

From the explicit formula given in (4.3) we get the exponential generating function of the $t$-successive associated $r$-Whitney numbers.

Theorem 10. The exponential generating function of the $t$-successive associated $r$-Whitney numbers is

$$
W_{k}^{[t]}(x):=\sum_{n \geq t k} W_{m, r}^{[t]} \frac{x^{n}}{n !}=\sum_{j=0}^{k}\left(\begin{array}{l}
k \\
j
\end{array}\right) \frac{(-1)^{k-j}}{k ! m^{k}} \frac{e^{(j m+r) x}}{(j m+r)^{(t-1) k}} .
$$

Corollary 6. For the 2-successive associated $r$-Whitney numbers,

$$
W_{k}^{[2]}(x)=\sum_{j=0}^{k}\left(\begin{array}{l}
k \\
j
\end{array}\right) \frac{(-1)^{k-j}}{k ! m^{k}} \frac{e^{(j m+r) x}}{(j m+r)^{k}} .
$$

These two result are more specified expressions as relation (2.9) of Theorem 4.

Proof. (Theorem 10) We use the derivation $(t-1) k$ times according to $x$ and using the consequence property, we get

$$
\begin{aligned}
\frac{\partial^{(t-1) k}}{\partial^{(t-1) k} x} W_{k}(x) & =\sum_{n \geq t k-(t-1) k} W_{m, r}^{[t]}(n+k(t-1), k) \frac{x^{n}}{n !} \\
& =\frac{1}{k ! m^{k}} e^{r x}\left(e^{m x}-1\right)^{k} \\
& =\frac{1}{k ! m^{k}} e^{r x} \sum_{j=0}^{k}\left(\begin{array}{l}
k \\
j
\end{array}\right)(-1)^{k-j} e^{j m x} \\
& =\sum_{j=0}^{k}\left(\begin{array}{l}
k \\
j
\end{array}\right) \frac{(-1)^{k-j}}{k ! m^{k}} e^{(j m+r) x} .
\end{aligned}
$$


Theorem 11. For $n \geq k$, we have

$$
W_{m, r}^{[t]}(n+(t-1) k, k)=\sum_{\substack{i_{1}+\cdots+i_{n}=n-k \\ i_{1}, \cdots, i_{n} \in\{0,1\}}} \prod_{j=0}^{n-1}\left(r+m\left(j-\sum_{\ell=1}^{j} i_{\ell}\right)\right)^{i_{j+1}} .
$$

Proof. By induction over $n$, we suppose that the identity is true until $n-1$

$$
\begin{aligned}
& \sum_{\substack{i_{1}+\cdots+i_{n}=n-k \\
i_{1}, \cdots, i_{n} \in\{0,1\}}} \prod_{j=0}^{n-1}\left(r+m\left(j-\sum_{l=1}^{j} i_{l}\right)\right)^{i_{j}+1} \\
= & \sum_{\substack{i_{1}+\cdots+i_{n-1}=(n-1)-(k-1) \\
i_{1}, \cdots, i_{n-1} \in\{0,1\}}} \prod_{j=0}^{n-2}\left(r+m\left(j-\sum_{l=1}^{j} i_{l}\right)\right)^{i_{j+1}} \\
& +\left(\begin{array}{c}
\substack{j \\
i_{1}+\cdots+i_{n-1}=(n-1)-k j=0 \\
i_{1}, \cdots, i_{n-1} \in\{0,1\}} \\
\sum_{l=1}^{n-2}\left(r+m\left(j-\sum_{l}\right)\right)^{i_{j+1}}
\end{array}\right)(r+m k) .
\end{aligned}
$$

We have

$$
\begin{aligned}
W_{m, r}^{[t]}(n & +(t-1) k, k) \\
& =(m k+r) W_{m, r}^{[t]}(n-1+(t-1) k, k)+W_{m, r}^{[t]}(n-1+(t-1)(k-1), k),
\end{aligned}
$$

which gives the desired result.

Corollary 7. For $n \geq t k$, we have

$$
W_{m, r}^{[t]}(n, k)=\sum_{\substack{i_{1}+\cdots+i_{n-(t-1) k}=n-t k \\ i_{1}, \cdots, i_{n-(t-1) k} \in\{0,1\}}} \prod_{j=0}^{n-(t-1) k-1}\left(r+m\left(j-\sum_{\ell=1}^{j} i_{\ell}\right)\right)^{i_{j}+1},
$$

with empty sum equal zero.

Example 1. For $k=t=2$ we have the following formula

$$
\begin{aligned}
W_{m, r}^{[2]}(n, 2)= & \sum_{i_{1}+\cdots+i_{n-2}=n-4} r^{i_{1}}\left(r+m\left(1-i_{1}\right)\right)^{i_{2}}\left(r+m\left(2-i_{1}-i_{2}\right)\right)^{i_{3}} \times \\
& \cdots \times\left(r+m\left(n-3-i_{1}-i_{2}-\cdots-i_{n-3}\right)\right)^{i_{n}-2}, \\
W_{m, r}^{[2]}(4,2)= & \sum_{i+j=0} r^{i}(r+m(1-i))^{j}=1,
\end{aligned}
$$




$$
\begin{aligned}
W_{m, r}^{[2]}(5,2) & =\sum_{i+j+k=1} r^{i}(r+m(1-i))^{j}(r+m(2-i-j))^{k}=3(r+m), \\
W_{m, r}^{[2]}(6,2) & =\sum_{i+j+k+\ell=2} r^{i}(r+m(1-i))^{j}(r+m(2-i-j))^{k}(r+m(3-i-j-k))^{\ell} \\
& =6 r^{2}+12 m r+7 m^{2} .
\end{aligned}
$$

Theorem 12. We have the following explicit formula

$$
W_{m, r}^{[t]}(n+t k, k)=\frac{1}{m^{k} k !} \sum_{j=0}^{k-j}(-1)^{k-j}\left(\begin{array}{l}
k \\
j
\end{array}\right)(m j+r)^{n+k},
$$

and thus

$$
W_{m, r}^{[t]}(n, k)=\frac{1}{m^{k} k !} \sum_{j=0}^{k}(-1)^{k-j}\left(\begin{array}{l}
k \\
j
\end{array}\right)(m j+r)^{n-(t-1) k} .
$$

Proof. It suffices to set $u_{k}=m k+r$ then $\left[u_{j}-u_{i}=m(j-i)\right]$ in Theorem 3 .

For $t=2$ we get Theorem 7 .

Theorem 13. Expression of $t$-successive $r$-Whitney numbers in terms of binomials and Stirling numbers.

$$
\left.W_{m, r}^{[t]}(n+t k, k)=\left(\frac{r}{m}\right)\right)^{k} \sum_{i=0}^{n+k}\left(\begin{array}{c}
n+k \\
i
\end{array}\right) r^{n-i} m^{i}\left\{\begin{array}{l}
i \\
k
\end{array}\right\} .
$$

Proof.

$$
\begin{aligned}
W_{m, r}^{[t]}(n+t k, k) & =\frac{1}{m^{k} k !} \sum_{j=0}^{k} \sum_{i=0}^{n+k}(-1)^{k-j}\left(\begin{array}{c}
k \\
j
\end{array}\right)\left(\begin{array}{c}
n+k \\
i
\end{array}\right)(m j)^{i} r^{n+k-i} \\
& =\frac{1}{m^{k} k !} \sum_{i=0}^{n+k} m^{i} r^{n+k-i}\left(\begin{array}{c}
n+k \\
i
\end{array}\right) \sum_{j=0}^{k}(-1)^{k-j}\left(\begin{array}{c}
k \\
j
\end{array}\right) j^{i} \\
& =\frac{1}{m^{k}} \sum_{i=0}^{n+k} m^{i} r^{n+k-i}\left(\begin{array}{c}
n+k \\
i
\end{array}\right)\left\{\begin{array}{c}
i \\
k
\end{array}\right\} .
\end{aligned}
$$

Notice that $\sum_{j=0}^{k}(-1)^{k-j}\left(\begin{array}{c}k \\ j\end{array}\right) j^{i}=0$ for $i<k$.

5. A $q$-ANALOGUE Of THE $t$-SUCCESSIVE ASSOCIATED STIRLING NUMBERS

Finally, we considerer a $q$-analogue of the $t$-successive associated Stirling numbers of the second kind. For this purpose, we use a similar statistic studied by Carlitz [6], see also [21]. 
Let $\pi=B_{1} / B_{2} / \cdots / B_{k}$ be any block representation of a set partition in $\Pi_{0,1}^{[t]}(n, k):=$ $\Pi^{[t]}(n, k)$, with $\min \left(B_{1}\right)<\min \left(B_{2}\right)<\cdots<\min \left(B_{k}\right)$. We define the following statistic on the set $\Pi^{[t]}(n, k)$.

$$
w^{[t]}(\pi):=\sum_{i=1}^{k}(i-1)\left(\left|B_{i}\right|-t+1\right) .
$$

We now define the q-analogue of the $t$-successive associated Stirling numbers of the second kind.

Definition 1. Define $\left\{\begin{array}{l}n \\ k\end{array}\right\}_{q}^{[t]}$ as the distribution polynomial for the $w^{[t]}$ statistic on the set $\Pi^{[t]}(n, k)$, that is,

$$
\left\{\begin{array}{l}
n \\
k
\end{array}\right\}_{q}^{[t]}=\sum_{\pi \in \Pi^{[t]}(n, k)} q^{w^{[t]}(\pi)}, \quad n, k \geq 0,
$$

where $q$ is an indeterminate.

It is clear that $\left\{\begin{array}{l}n \\ k\end{array}\right\}_{1}^{[t]}=\left\{\begin{array}{l}n \\ k\end{array}\right\}^{[t]}$.

For example, in the set $\Pi^{[2]}(7,3)$ we have the following partitions:

$\{\{1,2\},\{3,4,5\},\{6,7\}\}, \quad\{\{1,2,3\},\{4,5\},\{6,7\}\}, \quad\{\{1,2,5\},\{3,4\},\{6,7\}\}$,

$\{\{1,2\},\{3,4\},\{5,6,7\}\}, \quad\{\{1,2\},\{3,4,7\},\{5,6\}\}, \quad\{\{1,2,7\},\{3,4\},\{5,6\}\}$.

Therefore,

$$
\left\{\begin{array}{l}
7 \\
3
\end{array}\right\}_{q}^{[2]}=q^{4}+q^{3}+q^{3}+q^{5}+q^{4}+q^{3}=3 q^{3}+2 q^{4}+q^{5} .
$$

Let us introduce the following notations.

$$
[n]_{q}=1+q+\cdots+q^{n-1}, \quad[n]_{q} !=[1]_{q}[2]_{q} \cdots[n]_{q} \quad \text { and } \quad\left[\begin{array}{l}
n \\
k
\end{array}\right]_{q}=\frac{[n]_{q} !}{[k]_{q} ![n-k]_{q} !} \text {. }
$$

The last coefficient is called $q$-binomial coefficient. If $q=1$, then $\left[\begin{array}{l}n \\ k\end{array}\right]_{1}=\left(\begin{array}{l}n \\ k\end{array}\right)$.

Theorem 14. For $n \geq t k$, we have

$$
\left\{\begin{array}{l}
n \\
k
\end{array}\right\}_{q}^{[t]}=[k]_{q}\left\{\begin{array}{c}
n-1 \\
k
\end{array}\right\}_{q}^{[t]}+q^{k-1}\left\{\begin{array}{l}
n-t \\
k-1
\end{array}\right\}_{q}^{[t]} .
$$

Proof. For any set partition of $\Pi^{[t]}(n, k)$, there are two options: either $n$ form a block with its $t-1$-predecessors, or $n$ is in a block that satisfies the conditions. In the first case, there are $q^{k-1}\left\{\begin{array}{c}n-t \\ k-1\end{array}\right\}_{q}^{[t]}$ possibilities. In this case, the size of the last block $B_{k}$ is $t$, then this block contributes a factor $q^{k-1}$. In the second case, 
the element $n$ can be place into one of the $k$ blocks and thus contributes a factor $1+q+q^{2}+\cdots+q^{k-1}=[k]_{q}$. Moreover, the remaining elements can be chosen in $\left\{\begin{array}{c}n-1 \\ k\end{array}\right\}_{q}^{[t]}$ ways. Altogether, we have $[k]_{q}\left\{\begin{array}{c}n-1 \\ k\end{array}\right\}_{q}^{[t]}$ possibilities.

From above theorem, we obtain the following corollaries.

Corollary 8. For $k \geq 1$,

$$
\sum_{n \geq t k}\left\{\begin{array}{l}
n \\
k
\end{array}\right\}_{q}^{[t]} x^{n}=\frac{x^{t k} q^{\left(\begin{array}{c}
k \\
2
\end{array}\right)}}{(1-x)\left(1-[2]_{q} x\right)\left(1-[3]_{q} x\right) \cdots\left(1-[k]_{q} x\right)} .
$$

Moreover, the q-analogue of the $t$-successive associated $r$-Stirling numbers are given by the explicit identity

$$
\left\{\begin{array}{l}
n \\
k
\end{array}\right\}_{q}^{[t]}=q^{\left(\begin{array}{c}
k \\
2
\end{array}\right)} \sum_{i_{1}+i_{2}+\cdots+i_{k}=n-t k}[1]_{q}^{i_{1}}[2]_{q}^{i_{2}} \cdots[k]_{q}^{i_{k}},
$$

for $n \geq t k$.

Corollary 9. For $n \geq t k$ we have

$$
\left\{\begin{array}{l}
n \\
k
\end{array}\right\}_{q}^{[t]}=\frac{1}{[k]_{q} !} \sum_{j=1}^{k}(-1)^{k-j}\left[\begin{array}{l}
k \\
j
\end{array}\right]_{q} q^{\left({ }^{k-j}\right)}\left([j]_{q}\right)^{n-(t-1) k} .
$$

\section{ACKNOWLEDGEMENT}

The authors would like to thank the anonymous referee for some useful comments.

\section{REFERENCES}

[1] H. Belbachir and I. E. Bousbaa, "Associated Lah numbers and $r$-Stirling numbers." arXiv:1404.5573, pp. 1-24, 2014.

[2] H. Belbachir and A. F. Tebtoub, "Les nombres de Stirling associés avec succession d'ordre 2, nombres de Fibonacci-Stirling et unimodalité." C. R., Math., Acad. Sci. Paris, vol. 353, no. 9, pp. 767-771, 2015, doi: doi.org/10.1016/j.crma.2015.06.008.

[3] H. Belbachir and A. F. Tebtoub, "The $t$-successive associated Stirling numbers, $t$-FibonacciStirling numbers, and unimodality." Turkish J. Math., vol. 41, no. 5, pp. 1279-1291, 2017, doi: doi.org/10.3906/mat-1506-83.

[4] M. Bóna and I. Mező, "Real zeros and partitions without singleton blocks.” Eur. J. Comb., vol. 51, pp. 500-510, 2016, doi: doi.org/10.1016/j.ejc.2015.07.021.

[5] A. Z. Broder, "The $r$-Stirling numbers." Discrete Math., vol. 49, pp. 241-259, 1984, doi: doi.org/10.1016/0012-365X(84)90161-4.

[6] L. Carlitz, Generalized Stirling numbers. Combinatorial Analysis Notes, Duke University, 1968.

[7] J. Y. Choi, L. Long, S.-H. Ng, and J. Smith, "Reciprocity for multirestricted Stirling numbers." J. Comb. Theory, Ser. A, vol. 113, no. 6, pp. 1050-1060, 2006, doi: doi.org/10.1016/j.jcta.2005.10.001. 
[8] J. Y. Choi and J. D. H. Smith, "On the combinatorics of multi-restricted numbers." Ars Comb., vol. 75, pp. 45-63, 2005.

[9] L. Comtet, "Nombres de Stirling généraux et fonctions symétriques." C. R. Acad. Sci., Paris, Sér. A, vol. 275, pp. 747-750, 1972.

[10] L. Comtet, Advanced combinatorics. $\quad$ D. Reidel Publishing Co. (Dordrecht, Holland), 1974.

[11] F. T. Howard, "Associated Stirling numbers." Fibonacci Quart., vol. 18, pp. 303-315, 1980.

[12] T. Komatsu, I. Mező, and L. Szalay, "Incomplete Cauchy numbers." Acta Math. Hung., vol. 149, no. 2, pp. 306-323, 2016, doi: 10.1007/s10474-016-0616-z.

[13] T. Komatsu, "Incomplete poly-Cauchy numbers." Monatsh. Math., vol. 180, no. 2, pp. 271-288, 2016, doi: 10.1007/s00605-015-0810-z.

[14] T. Komatsu, K. Liptai, and I. Mező, "Incomplete poly-Bernoulli numbers associated with incomplete Stirling numbers." Publ. Math., vol. 88, no. 3-4, pp. 357-368, 2016.

[15] T. Komatsu and J. L. Ramírez, "Generalized poly-Cauchy and poly-Bernoulli numbers by using incomplete $r$-Stirling numbers." Aequationes Math., vol. 91, no. 6, pp. 1055-1071, 2017, doi: 10.1007/s00010-017-0509-4.

[16] T. Komatsu and J. L. Ramírez, "Incomplete poly-Bernoulli numbers and incomplete poly-Cauchy numbers associated to the $q$-Hurwitz-lerch zeta function." Mediterr. J. Math., vol. 14, no. 3, p. 19, 2017, doi: 10.1007/s00009-017-0935-5.

[17] I. Mező, "A new formula for the Bernoulli polynomials." Result. Math., vol. 58, no. 3-4, pp. 329-335, 2010, doi: 10.1007/s00025-010-0039-z.

[18] I. Mező, "Periodicity of the last digits of some combinatorial sequences." J. Integer Seq., vol. 17, no. 1 , pp. article 14.1.1, 18, 2014.

[19] I. Mezô and J. L. Ramírez, "The linear algebra of the $r$-Whitney matrices.” Integral Transforms Spec. Funct., vol. 26, no. 3, pp. 213-225, 2015, doi: 10.1080/10652469.2014.984180.

[20] V. H. Moll, J. L. Ramírez, and D. Villamizar, "Combinatorial and arithmetical properties of the restricted and associated Bell and factorial numbers." J. Comb., vol. 9, no. 4, pp. 693-720, 2018, doi: 10.4310/JOC.2018.v9.n4.a7.

[21] C. G. Wagner, "Partition statistics and $q$-Bell numbers $(q=-1)$." J. Integer Seq., vol. 7, no. 1, pp. art. 04.1.1, 12, 2004.

Authors' addresses

Hacène Belbachir

USTHB, Faculty of Mathematics, RECITS Laboratory. P.B. 32, El Alia, 16111, Bab Ezzouar, ALGERIA

E-mail address: hbelbachir@usthb.dz; hacenebelbachir@gmail.com

Carolina Forero

Universidad Sergio Arboleda, Departamento de Matemáticas, Bogotá, COLOMBIA

E-mail address: tcarolinafc@gmail.com

José L. Ramírez

Universidad Nacional de Colombia, Departamento de Matemáticas, Bogotá, COLOMBIA

E-mail address: jlramirezr@unal.edu.co 